\title{
NOTE
}

\section{Synthesis and Release of Prolactin and GH in vitro from the Anterior Pituitaries of Virgin or Pregnant Mice}

\author{
REIKo YANAI AND HiRoshi NAGASAWA \\ Pharmacology Division, National Cancer Center Research Institute, \\ Tsukiji 5-1-1, Chuo-ku, Tokyo
}

\begin{abstract}
Synopsis
The secretory activities of prolactin and $\mathrm{GH}$ of the mouse anterior pituitary were determined from ${ }^{14} \mathrm{C}$-leucine incorporation in vitro into these hormones. Prolactin and GH of the mouse anterior pituitary were fractionated by disc electrophoresis on polyacrylamide gel.

Neither synthesis nor release of prolactin and $\mathrm{GH}$ was different between estrus and diestrus in $\mathrm{C} 3 \mathrm{H} / \mathrm{He}$ strain of virgin mice. No relation existed between the number of placentae and the pituitary secretory activities of these hormones on day 19 of pregnancy when the number of placentae was adjusted to 1-8 each on day 8. Synthesis of prolactin and GH was significantly higher and percent release of these hormones was lower on day 19 of pregnancy than during virginal stages.
\end{abstract}

Recently, hormone secretory activities of the anterior pituitary have been estimated fairly precisely by measuring the serum or plasma levels of these hormones in some species using the radioimmunoassay technique. In the mouse, $\mathrm{GH}$ in plasma has been able to be measured by applying the double antibody radioimmunoassay for rat $\mathrm{GH}$ (Müller et al., 1971a, b), but the determination of serum prolactin is still impossible.

Yamamoto et al. (1970) reported that the rate of synthesis and release of prolactin and $\mathrm{GH}$ from the rat anterior pituitary could be determined by measuring in vitro incorporation of ${ }^{14} \mathrm{C}$-leucine after fractionating these hormones in the pituitary and medium by polyacrylamide disc electrophoresis. The mouse prolactin and $\mathrm{GH}$ are also detected by disc electrophoresis by varying the concentration of acrylamide to $10 \%$ and both hormone bands were identified using bioassay (Cheever et al., 1969; Lewis et al., 1969). These

Received for publication January 8, 1972. works prompted us to investigate whether or not the evaluation of the pituitary function to synthesize or release prolactin and $\mathrm{GH}$ was possible in mice by applying the technique of Yamamoto et al. (1970). The pituitary prolactin contents were not different between estrus and diestrus in mice (Yanai and Nagasawa, 1971). In rats, however, Amenomori et al. (1970) found that the serum prolactin level was significantly higher at estrus than at diestrus, whereas pituitary contents were not significantly different between these stages and that it was lower during pregnancy than at diestrus, while it rose at the term of pregnancy. In the mouse, we confirmed that the pituitary prolactin concentration decreased with the advance of pregnancy (Yanai and Nagasawa, 1971). During the latter half of pregnancy, the placentae are generally supposed to be the primary sources of prolactin- and GH-like substances (Amoroso and Porter, 1966). It is, therefore, interesting to study by the technique of Yamamoto et al. (1970) the difference in pituitary secretory 
activities of these hormones between estrus and diestrus and to study the effects of number of placentae on these pituitary functions in order to know the pituitary physiology during pregnancy in mice. The present experiments deal with these problems.

\section{Materials and Methods}

\begin{abstract}
Animals
Seventy to 80 day old $\mathrm{C} 3 \mathrm{H} / \mathrm{He}$ strain of female mice bred in our laboratory were used. They were maintained in an airconditioned and artificially illuminated room and provided with commercial diet and water ad libitum. Some mice showing normal estrous cycles were killed by decapitation at estrus or at diestrus and the anterior pituitaries were used for incubation. The others were mated with males and the day when copulation plug or sperms were found or the last estrous day was designated as day 1 of pregnancy. The fetuses and placentae of the pregnant mice were adjusted to 1-8 each by laparotomy on day 8 of pregnancy. On day 19 of pregnancy, they were killed and the anterior pituitaries were incubated.
\end{abstract}

\section{Incubation}

The incubation procedure was fundamentally the same as reported by Yamamoto et al. (1970). However, the volume of the medium was $0.15 \mathrm{ml}$ per tube containing a single mouse anterior pituitary, and the incubation time was $4 \mathrm{hr}$. The incubation medium was Ringer bicarbonate buffer solution ( $\mathrm{pH}$ 7.4). Three hundred and sixty $\mu \mathrm{g}$ of glucose and $0.1 \mu \mathrm{Ci}$ of ${ }^{14} \mathrm{C}$-leucine $(311 \mathrm{mCi} / \mathrm{mmole}, \quad 50 \mu \mathrm{Ci} / \mathrm{ml}$, the Radiochemical Centre, Amersham, England) were added to $0.2 \mathrm{ml}$ of the medium. After incubation, the incubated tissue was taken out of the medium, washed with ice-cold $0.9 \%$ saline, and homogenized in $0.15 \mathrm{~m} l$ of distilled water. "Cold"' anterior pituitary homogenate was added to the incubated tissue homogenate as "carrier". These mixtures were kept in a refrigerator.

\section{Disc electrophoresis}

Pituitary proteins in the homogenate and the medium were fractionated by disc electrophoresis. The separating gel contained $10 \%$ acrylamide at $\mathrm{pH}$ 9.5. The homogenate and medium were mixed with an equal volume of twice concentrated stacking gel and $3 / 4$ of the mixture was polymerized as sample gel. After electrophoresis, the separating layer was stained in $0.002 \%$ amidoblack. Prolactin and $\mathrm{GH}$ bands were visible in $10 \mathrm{~min}$, and both bands were cut out and prepared for liquid scintillation counting (Yamamoto et al., 1970). The value in the medium represents released hormone and the summation of the values in the pituitary and the medium represents the total synthesis of the hormone. The rate of synthesis and release of prolactin and $\mathrm{GH}$ was expressed in terms of $\mathrm{cpm}$ of incorporated ${ }^{14} \mathrm{C}$-leucine into prolactin or GH per mouse pituitary.

Relation between the number of anterior pituitaries and the incorporation of ${ }^{14} \mathrm{C}$-leucine into the hormone

This was performed in order to check the linearity between the number of pituitary and its hormone secretory activity. Eight pituitaries of old female mice were incubated in $0.3 \mathrm{ml}$ of the medium for $4 \mathrm{hr}$. After incubation, the pituitaries were homogenized with $0.4 \mathrm{~m} l$ of distilled water. Both the medium and the homogenate were divided into three parts equivalent to 4,2 and 1 pituitaries and each solution was applied to disc electrophoresis.

\section{Results}

The relations between the number of anterior pituitaries and the radioactivities of synthesized and released prolactin and GH are presented in Figure 1. The linear relations between these items were observed both in prolactin and in $\mathrm{GH}$.

Figure 2 shows the radioactivity of pituitary prolactin synthesized or released and the percent release during virginal stages and on day 19 of pregnancy in mice with different numbers of placentae. Both pituitary prolactin synthesis and release were not different between estrus and diestrus. No relation was obtained between the number of placentae and the pituitary prolactin secretion. The mean value of prolactin synthesized on day 19 of pregnancy was significantly higher than that at estrus or diestrus $(\mathrm{P}<0.01)$. On the other hand, the percent release of prolactin was significantly lower on day 19 of pregnancy than during the virginal stages $(\mathrm{P}<0.05)$.

Similar results were observed in GH (Fig. 3). Not only pituitary GH synthesis but also release was not different between estrus and 


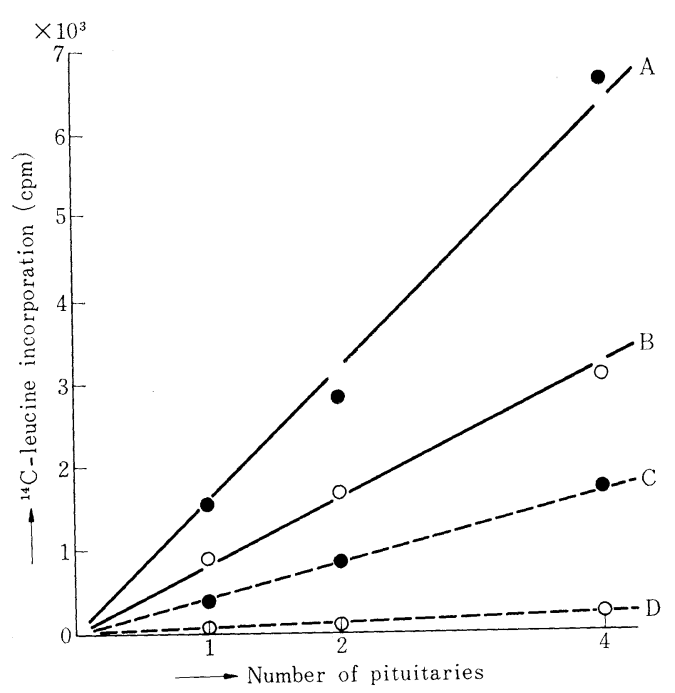

Fig. 1. Relations between the number of mouse anterior pituitaries incubated and the synthesis and release of prolactin and $\mathrm{GH}$.
A: Prolactin synthesis.
B: GH synthesis.
$\mathrm{C}$ : Prolactin release.
D: GH release.

diestrus. Synthesis and release of GH were not influenced at all by the number of placentae on day 19 of pregnancy. The mean value of GH synthesized on day 19 of pregnancy was significantly higher than those during virginal stages. Therefore, the percent release of $\mathrm{GH}$ was significantly lower on day 19 of pregnancy than during virginal stages $(\mathrm{P}<$ 0.01).

\section{Discussion}

The linear relations between the number of anterior pituitaries and radioactivities of both prolactin and GH synthesized and released would indicate that the method of Yamamoto et al. (1970) in rats was applicable to mice.

In the present experiments, the pituitary functions of prolactin and GH synthesis and release in mice were not significantly different between estrus and diestrus. In rats, Ieiri et al. (1971) found by the same technique that the synthesis, release and percent release of prolactin were significantly higher at estrus than at diestrus, while those of $\mathrm{GH}$ were not different among the stages of estrous cycle. In this respect, Müller et al. (1971a) demonstrated that insulin hypoglycemia and cold exposure highly effective in the rat to release pituitary GH were unable to increase mouse plasma GH levels, and suggested that the mouse was a species in which the GH secretion was already maximal in basal conditions due to the very elevated metabolic rate and could not be further increased by any of the stimuli that were highly active in other species. This may partly account for the discrepancy in the pituitary prolactin and GH secretory activities between rats and mice.

The pregnancy is maintained and mammary growth proceeds normally by placental mammotropin (prolactin-like substance) during the latter half of pregnancy (Amoroso and Porter, 1966). Contopoulos and Simpson (1959) reported that there was a threefold increase in growth-promoting potency in rat plasma during pregnancy, which was not abolished by hypophysectomy. The present results show that the number of placentae never influences pituitary functions of prolactin and GH synthesis and release. The percent release of both prolactin and $\mathrm{GH}$ was significantly lower on day 19 of pregnancy than during the virginal stages despite the significantly higher synthesis. This may speculate the existence of negative feedback by placental prolactin-like and GH-like substances. But further investigations are necessary for obtaining the definite conclusion.

In the present experiments, the radioactivities of hormones synthesized and released per pituitary were lower in Figure 1 than in both Figure 2 and Figure 3. This may be due to the volume of incubation medium per pituitary, which in the former was $1 / 4$ of that in either of the latters. It is likely that the more the volume of the medium per unit weight of pituitary is, the more active the pituitary functions are. 

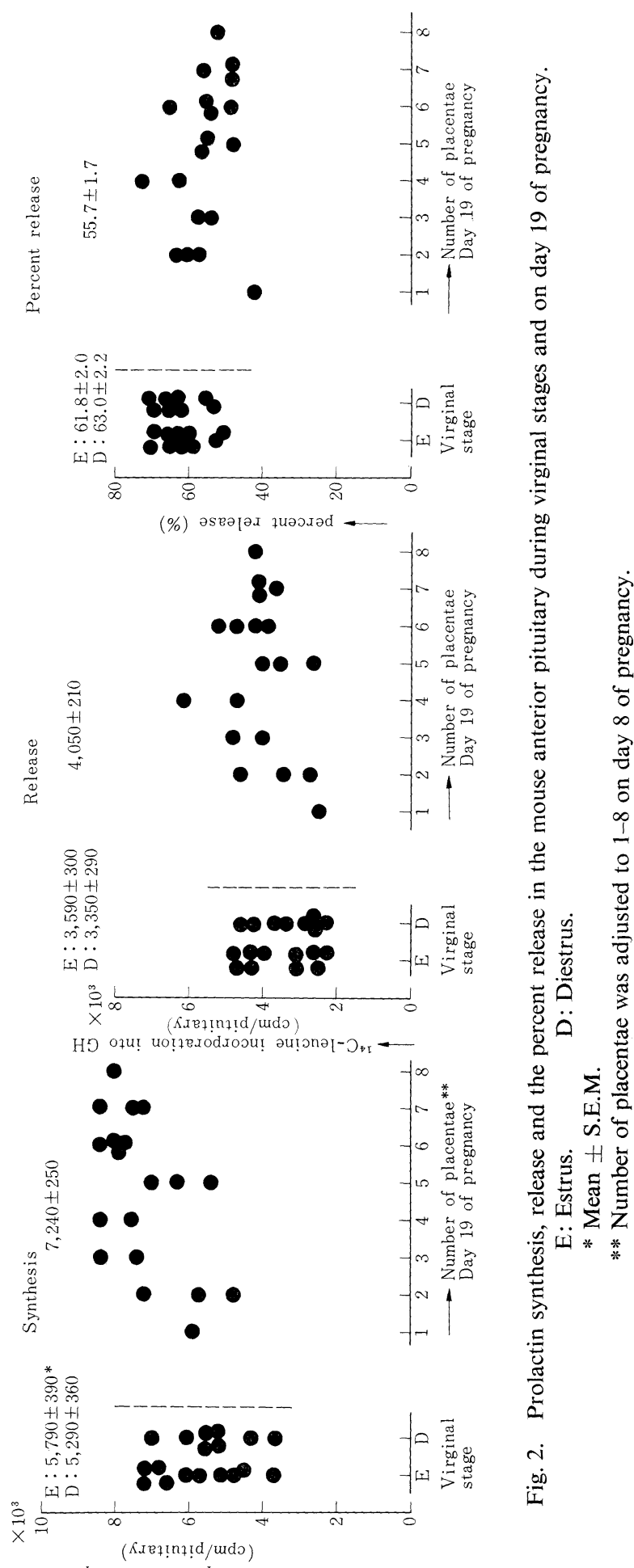

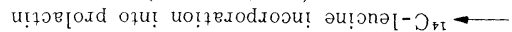




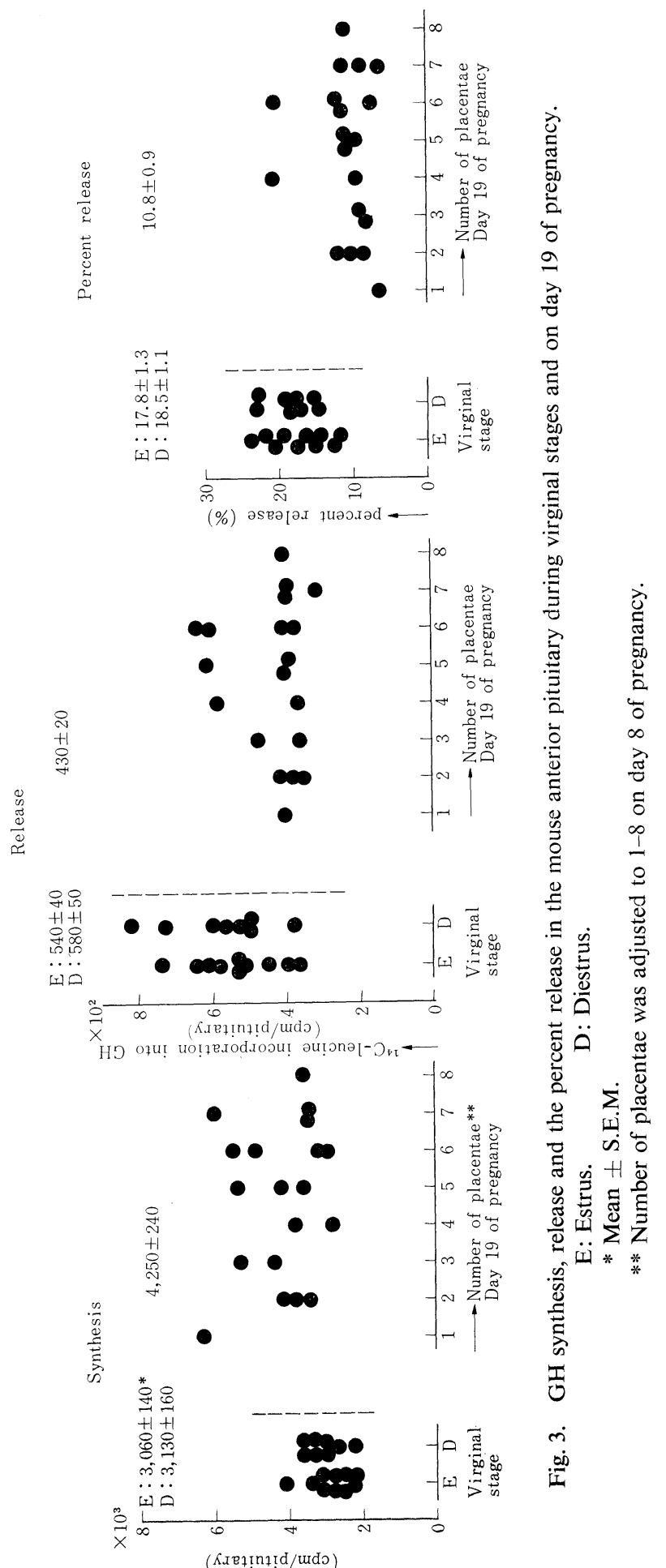

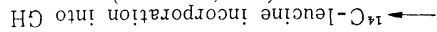




\section{Acknowledgements}

We wish to express our gratitude to Prof. K. Yamamoto and Dr. T. Ieiri, Institute of Endocrinology, Gumma University, Maebashi, for their valuable advice and suggestion.

\section{References}

Amenomori, Y., C. L. Chen and J. Meites (1970). Endocrinology 86, 505.

Amoroso, E. C. and D. G. Porter. "Pituitary Gland" (Harris, G. W. and B. T. Donovan eds.). Vol. 2. Butterworth, London. Chap. 12, p. 364 (1966).

Cheever, E. V., B. K. Seavey and U. J. Lewis
(1969). Endocrinology 85, 698.

Contopoulos, A. N. and M. E. Simpson (1959). Ibid. 64, 1023.

Contopoulos, A. N. and M. E. Simpson (1959). Ibid. 64, 1023.

Ieiri, T., Y. Akikusa and K. Yamamoto (1971). Ibid. 89, 1533.

Lewis, U. J., M. Litteria and E. V. Cheever (1969). Ibid. 85, 690.

Müller, E. E., D. Miedico, G. Giustina and D. Cocchi (1971a). Ibid. 88, 345.

Müller, E. E., D. Miedico, G. Giustina, A. Pecile, D. Cocchi and V. Mandelli (1971b). Ibid. 89, 56.

Yamamoto, K., L. M. Taylor and F. E. Cole Cole (1970). Ibid. 87, 21.

Yanai, R. and H. Nagasawa (1971). Horm. Behav. 2, 73. 